AperTO - Archivio Istituzionale Open Access dell'Università di Torino

\title{
Adjuvant Treatment of Melanoma: Recent Developments and Future Perspectives
}

\section{This is a pre print version of the following article:}

Original Citation:

\section{Availability:}

This version is available http://hdl.handle.net/2318/1717982

since 2019-11-30T00:09:03Z

Published version:

DOI:10.1007/s40257-019-00456-4

Terms of use:

Open Access

Anyone can freely access the full text of works made available as "Open Access". Works made available under a Creative Commons license can be used according to the terms and conditions of said license. Use of all other works requires consent of the right holder (author or publisher) if not exempted from copyright protection by the applicable law. 
Adjuvant treatment of melanoma - recent developments and clinical trial outcomes

Alessandro Testori ${ }^{1 *}, \mathrm{MD}$, Simone Ribero ${ }^{2^{*}}, \mathrm{MD}, \mathrm{PhD}$ and Mario Mandalà ${ }^{3}, \mathrm{MD}$

1.Chairman surgical subgroup, EORTC melanoma group, EORTC, Brussel, BE

2.Medical Sciences Department, Dermatologic clinic, University of Turin, Turin, Italy

3.Unit of Medical Oncology, Department of Oncology and Hematology, Papa Giovanni XXIII Hospital, Bergamo, Italy

running title Adjuvant treatment of melanoma

*equal contribution

Corrispondance to

XXXXX

\begin{abstract}
For early melanoma, surgical excision is the treatment of choice and this strategy is initially curative for the majority of patients. However, only approximately $40-60 \%$ of patients who have surgery alone and higher risk stages, will be disease-free after 5 years of follow up, depending on the original III stage of the disease. These patients will relapse either with locoregional or disseminated disease. Adjuvant therapies are required to be able to reduce the recurrence rate on radically operated patients in these different initial stages of the disease.

New treatments have appeared in the landscape of metastatic melanoma and this have opened to new potential scenarios in the adjuvant setting. In particular immunotherapy, immunocheckpoint inhibitors and target therapies have been recently published their potential advantage from the results obtained in the curative setting for stage IV, where the different mechanisms of action could even be potentially more active and more responsive due to the limited subclinical presence of disease in the patients after surgical complete resection.
\end{abstract}


Currently, interferon alfa (IFN), ipilimumab and more recently antipd1 are immunotherapeutic options are approved for adjuvant treatment of melanoma in US, while in EU only IFN is for clinical use. Other adjuvant treatments have been published and are currently in the phase of approval trough FDA and EMA, based on the results of clinical trials that include PD-1 inhibitors and small-molecule BRAF+ MEK inhibitors.

Actually the first study designed to answer this question is the Keynote 054 (pembrolizumab (MK-3475) versus Placebo after complete resection of high stage III Melanoma) which, through a cross over plan on recurring patients, will be able to define if patients treated in the adjuvant setting will describe a better survival compared to patients treated after recurrence.

A completely new scenario will also become evident from the opportunity to open the therapeutic approach from a neoadjuvant setting: since new therapies are available, patients with macroscopic nodal metastases so far considered operable, might be sent to a medical approach in stage III and surgery proposed only as final resource both to remove disease residuals and to confirm the efficacy of the treatment, while in advanced stage III/IV not operable at the diagnosis of an initially disease advanced situation, patients may partially respond to the new therapies and after obtaining a partial/complete reduction of the disease, become virtually operable from the surgical point of view.

\section{Introduction}

Melanoma accounts for a small percentage of all skin malignancies, but it is responsible for the majority of deaths due to skin cancers worldwide ${ }^{1}$. Moreover, cause of the increasing aging of the population, the age at death of melanoma patients has steadily increased, with present predictions showing that the number of melanoma cases will increase. Due to the introduction of new systemic drugs, we assist to an increase survival for advanced, unresectable and metastatic melanoma over the last few years. This unprecedented development is related to the introduction of immune checkpoint inhibitors with antibodies against CTLA-4 and PD-1 and targeted therapy with BRAF and MEK inhibitors.

The recent developments and approvals in immunotherapy and targeted agents that have significantly changed the landscape of melanoma therapy in the metastatic setting can represent a great promise for adjuvant and neoadjuvant treatment in high-risk or advanced locoregional disease. 
All adjuvant approaches had to be initially tested on an advanced disease therapeutic approach. This review of historical and recent drugs is willing to report on the situation we are facing at the moment where we stand in front of a new era in the therapeutical approach of advanced melanoma patients which is the basis of novel approaches in the adjuvant settings as recent studies come to publication and start their approval pathways through regulatory agencies Worldwide. In this review for an easier comparison between old and recent studies we will consider the 7 th American Joint Comitee on cancer (AJCC) ${ }^{2}$ and not the $8^{\text {th }} 34$.

\section{STAGE II-III}

Melanoma patients with intermediate and thick tumours are offered a sentinel node biopsy (SLNB) to identify lymph node spread as this procedure has prognostic value, stratifying patients in different risk categories ${ }^{5}$. Other parameters such as mitoses and ulceration are also helpful in thinner melanomas ${ }^{6}$. After a positive SLN, the current guidelines recommended a complete lymph node dissection (CLND) of all the involved metastatic basins but in selected patients (to be defined if with very small deposits in the lymph node, as for breast cancer patients or with macroscopic disease due to a virtual situation of microscopic subclinical advanced disease ${ }^{7}$ ) may be given the choice of avoiding a lymphadenectomy. The final results of the DecoG and MSLT II trials ${ }^{8,9}$ may be useful to propose new biologically driven guide lines on $\mathrm{N}+$ patients, but a careful discussion will be needed once all data are supported by longer follow up periods: so far the published data on these 2 trials do not show any survival benefit for the patients undergoing a CLND after the diagnosis of metastatic SLN, but only a reduced risk of nodal locoregional relapse for the patients immediately operated. On the contrary if the subgroups analysis on MSLT II study is brought to a speculative discussion, the opposite seems to be more rational: patients with microscopic deposits in the SLN could benefit from a CLND, while patients with macrometastases would reach the same OS whether operated or not with an immediate CLND. The biological explanation of this different behaviour can be related to the fact that a certain percentage of patients with micrometastases could only have few tumoral cells into the nodes 
that are removed with a CLND, while in case of macrometastases, the disease may have also spread heamatogenously, making totally un-useful the proposed CLND.

The number of positive lymph nodes (LN) and its ratio represent the two most important prognostic factors in stage III melanoma patients ${ }^{10,11}$. The 5 -year survival of melanoma patients with LN metastasis ranges from an average of $20-40 \%$ when patients present with clinically evident nodal disease, improving to $67 \%$ when patients had their LN metastasis identified with SLNB. Patients who have CLND after a positive SLNB show a wide heterogeneity in their

prognosis, with 5-year survival rates ranging from 15\% in case of multiple positive LNs to $90 \%$ in case of a small cellular metastatic deposits in the SLN where the prognosis is even more favorable then that of high risk stage II patients $(>4 \mathrm{~mm}$ Breslow, ulcerated primary melanoma pts.).

The risk of recurrence in stage III patients is very wide, and no available biomarker for predicting recurrence have been established so far. The best predictor of recurrence is the number of LN involved. Different nomograms based on clinical pathological features have been proposed for predicting which patients with positive SLN are more at risk of relapse ${ }^{12}$.

\section{BIOCHEMOTHERAPY}

Cisplatin and interleukin-2 (IL-2)-based biochemotherapy have been used for stage III melanoma. The trial on 432 high-risk patients assigned to either three cycles of cisplatin, vinblastine, dacarbazine, IL-2, and IFN- $\alpha$ given over a 9-week period or to high-dose IFN- $\alpha$ for 1 year. Results showed that the biochemotherapy regimen significantly prolonged RFS at a median follow-up of 7.2 years ${ }^{13}$. However, there was no significant difference in the OS (5-year rate $56 \%$ for both treatment arms; HR 0.98; 95\% CI, 0.74-1.31). The biochemotherapy regimen was substantially more toxic, with grade 3 or 4 side effects (consisting primarily of hematologic and gastrointestinal toxicity) observed in $76 \%$ of participants. Neurologic, psychiatric, and hepatic toxicities were the most frequent with high-dose IFN- $\alpha$. Biochemotherapy toxicities were limited to the 9-week treatment period, while IFN- $\alpha$ toxicities were distributed across the year of 
treatment. Even though the biochemotherapy regimen was the first to produce a significant improvement in RFS compared with an active control arm, the lack of OS benefit coupled with the failure of this regimen to show an OS benefit relative to chemotherapy alone in patients with stage IV disease has limited its acceptance in the adjuvant setting. ${ }^{14}$

\section{Adjuvant $\mathrm{CT}$ with/without BCG/INF}

Hypothetically, non-detected melanoma micrometastases might be the cause of future relapses and/or may induce tumor tolerance in the host. Different clinical trials comparing patients treated after CLND with immunotherapy with interferon-alpha-2b (IFN- $\alpha-2 b)$, bacille Calmette-Guérin vaccine, dacarbazine, or a combination of the last two failed iun showing a higher survival ratein the treatment arm. ${ }^{151617}$

A regimen of IFN- $\alpha-2 b$ administered for 1 year at maximum tolerated doses was approved by the FDA in 1995 and later on by EMA for the adjuvant therapy of patients with high-risk (AJCC Stage IIB and III) melanoma: up to now this is still the only approved drug in Europe for melanoma patients in the adjuvant setting.

Adjuvant IFN- $\alpha$ therapies, which could induce TH1 anti-tumor responses, are based on these hypotheses and might be of benefit to some patients with possible micrometastases ${ }^{18}$. IFN- $\alpha$ directly inhibits the proliferation of melanoma cells. Moreover, IFN- $\alpha$ decreases intracellular and secretory levels of VEGF in melanoma cell lines ${ }^{19}$, thus reducing microvessel density around the tumour. It has been described to be able to promot tumor immunogenicity and enhances anti-tumor immunity. MHC class I Expression has been analysed by several studies on both melanoma and immune cells when stimulated with IFN- $\alpha^{20}$. The use of IFN- $\alpha$ as an adjuvant therapy in melanoma patients is based on the hypothesis that micrometastatic disease is the cause of future relapses and may induce tumor tolerance in the host. Unfortunately the global efficacy on overall survival is as low as $3 \%$. 
During more then 3 decades, low- (LDI), intermediate (IDI)- and high-dose (HDI) IFN- $\alpha$ regimens have been tested in randomized trials in the adjuvant setting ${ }^{21,22}$; these studies greatly differed also in terms of the therapy duration, route of administration and the type of IFNs used. As just mentioned, the most important discussion is clearly on the dosage: a significant impact on overall survival (OS) was only shown with the high-dose IFN- $\alpha 2 b$ intravenous regimen (HDI) when compared to observation only (US Intergroup trials E1684: median OS 3.82 vs 2.78 years, $\mathrm{p}=0.0237)$ and the GMK vaccine (E1694: OS HR=1.52; $\mathrm{P}=0.009)$.. The outcomes of the E1684 trial in 1995 led to the regulatory approval of IFN by the US Food and Drug Administration (FDA). However, these results were not confirmed in the following E1690 trial that compared HDI versus LDI versus control. In fact it failed in demonstrating a significant benefit of the HDI, but bearing more adverse events ${ }^{23}$. Furthermore, different randomized trials reported other conflicting results, never offering the real hint to the therapeutic benefit of IFN. The randomized phase III DeCOG trial compared LDI vs LDI plus dacarbazine vs observation in stage III melanoma patients. The Authors found a DFS and OS for the LDI regimen, and, interestingly, a worse therapeutic effect when dacarbazine was added ${ }^{24}$.

The EORTC 18952 adjuvant IFN trial was designed to investigate also if an antiangiogenic effect could be relevant in the potential benefit of adjuvant IFN ${ }^{25}$. In this trial researchers compared: I) a 4 weeks-induction phase using IFN at 10 million IU/m2/d for 5 days/week for 4 weeks, followed by a maintenance phase with 10 million IU three times a week for 12 months; II) 5 million IU 3 days/week for 24 months; III) observation alone. After the long median followup of 11 years, the only difference reported was the distant metastasis-free interval with an HR of 0.95 for the shorter maintenance group versus HR of 0.82 for the longer maintenance group $(\mathrm{p}=0.027)$.

A metanalysis found that IFN $\alpha$ slightly improved DFS (risk reduction=18\%) and OS (risk reduction $=11 \%$ ) in high-risk cutaneous melanoma patients, however, the different studies analysed did not show any differences between low and high dosages ${ }^{26}$. Wheatley et al. reported a 5 -year absolute benefit of about 3\%, with greater efficacy in patients where the primary tumor was ulcerated ${ }^{27}$.

Clinical trials comparing adjuvant HDI to ipilimumab (NCT01274338, NCT01708941, NCT02506153) or to pembrolizumab (NCT02506153) are ongoing, and the results are still pending. 
Pegylated IFN $\alpha$ (peg-IFN), which should have a longer half-life through IFN's covalent binding to polyethylene glycol, was tested in the European Organization for Research and Treatment of Cancer (EORTC) trial $18991^{28}$. The trial tested an induction dosage of subcutaneous peg-IFN at $6 \mu \mathrm{g} / \mathrm{kg} / \mathrm{week}$ for 8 weeks, followed by a maintenance dose of weekly subcutaneous injections at $3 \mu \mathrm{g} / \mathrm{kg}$ for up to 5 years. A rather slight improvement of the relapse free survival for the pegIFN was reported (7-year RFS rate: $39.1 \%$ versus $34.6 \%$ ) but authors did not find any differences in OS and distant metastasis-free survival between the treatment and the sole observation group. A pooled analysis of the EORTC trials 18952 and 18991 found that the primary tumor ulceration and the presence of only micrometastases as lymph nodal involvement, could be predictive of IFN efficacy ${ }^{29}$. Moreover, one study reported peg-IFN's association with higher rates of grade 3-4 Adverse Events (47.3\% versus 25.2\%; $\mathrm{p}<0.0001)$ and treatment discontinuations ( $54.3 \%$ versus $30.4 \%$ ) compared to IFNa ${ }^{30}$.

\section{VACCINE FOR ADJUVANT TREATMENT}

Dendritic cells (DC) are the most efficient antigen-presenting cells of the immune system due to their capacity to activate and prime naive $\mathrm{T}$ cells ${ }^{31}$. They play a fundamental role in anticancer immunotherapy due to their role in induction of antitumor immunity. DC can be generated ex vivo, activated, and loaded with tumor antigens before to be injected into the patients. ${ }^{32}$ The rationale to include DC vaccination in the adjuvant treatment in stage III patients is that high tumor load causes immune suppression by secretion of immunosuppressive cytokines, and attraction of regulatory $\mathrm{T}$ cells and myeloid derived suppressor cells in the tumor microenvironment. The clinical effectiveness of DC vaccination might be improved by increasing the number of antigens. The melanoma differentiation antigens gp100 and tyrosinase were previously selected due to their expression on melanoma cells and have shown to be capable of inducing functional cytotoxic $\mathrm{T}$ cells. ${ }^{33}$ However, recent findings show that tumorspecific mutations, leading to neoantigens, may drive potent antitumor responses ${ }^{34}$. Carreno and colleagues found that a DC vaccine with carefully selected patient-specific neoantigens, led to an increase in the breadth and diversity of melanoma neoantigen-specific $\mathrm{T}$ cells in peripheral blood samples of three stage IV melanoma patients ${ }^{35}$. As most mutated proteins are essentially unique to a tumor, personalized antigen selection might be beneficial in vaccination strategies. A great 
challenge will be the identification of the right immunogenic neoantigens, especially in stage III melanoma patients, since only a minimal amount of tumor material might be available ${ }^{36}$. For this reason, and in light of tumor heterogeneity, it might be preferable to combine commonly expressed melanoma differentiation antigens with patient-specific neoantigens in future DC vaccines.

7.Adjuvant vaccine with melanoma antigen GM-2 ganglioside

The GM2 ganglioside is an antigen expressed in the majority of melanomas. The GM2-KLH/QS21 vaccine induces high immunoglobulin $\mathrm{M}(\operatorname{Ig} \mathrm{M})$ and $\operatorname{IgG}$ antibody responses documented in early phase clinical trials at the EORTC melanoma group ${ }^{37}$. The EORTC 18961 trial compared the efficacy of GM2-KLH/QS-21 vaccination versus observation on high risk primary melanoma (with negative SLN). A total of 1,314 patients with a primary tumor $>1.50 \mathrm{~mm}$ in thickness were randomly assigned to GM2-KLH/QS-21 vaccination $(n=657)$ or observation $(n=657)$. Treatment consisted of subcutaneous injections once per week from week 1 to 4 , then every 3 months for the first 2 years and every 6 months during the third year. Relapse-free survival (RFS) was the primary endpoint. Secondary endpoints were distant metastasis-free survival (DMFS) and OS. After a median follow-up of 1.8 years, the trial was stopped at the second interim analysis for futility regarding RFS (hazard ratio $[\mathrm{HR}], 1.00 ; P=.99$ ) and detrimental outcome regarding OS $(\mathrm{HR}, 1.66 ; P=.02)$. After a median follow-up of 4.2 years, 400 relapses, nine deaths without relapse and a total of 236 deaths had been recorded. At 4 years, the vaccination arm showed a decreased RFS rate of $1.2 \%$ (HR, 1.03; 95\% CI, 0.84 to 1.25) and OS rate of $2.1 \%(\mathrm{HR}, 1.16 ; 95 \% \mathrm{CI}, 0.90$ to 1.51$)$. GM2-KLH/QS-21 vaccination does not improve outcome for patients with stage II melanoma and may induce immune tolerance to tumoral antigens with a consequent worsening of the prognosis. 


\section{MAGE -A3}

MAGE-A3 is expressed on approximately $60 \%$ of melanoma specimens as a tumour specific protein. The DERMA (ADjuvant ImmunothERapy with MAGE3 in MelanomA) is a doubleblind, randomized, placebo-controlled phase III study of recombinant MAGE-A3 with AS15 antigen-specific cancer immunotherapeutic (ASCI) in stage IIIB/C patients with MAGE-A3positive. The study was based on an EORTC phase II study of patients with stage IV M1A disease that identified a superior survival benefit for patients receiving the MAGE-A3 vaccine treated with the AS15 rather than the ASO2B adjuvant (HR 0.55; 95\% CI, 0.28-1.06) ${ }^{38}$. Furthermore, a genetic predictor identified a group of patients receiving MAGE-A3 with AS15 ASCI with a better OS (HR 0.27; 95\% CI, 0.08-0.89). The DERMA study screened 3,914 patients and randomly assigned 1,344 patients 2:1 to 13 intramuscular injections of vaccine or placebo. The latter failed to meet its coprimary endpoint of DFS in either the overall population of patients studied or in those with the potential predictive gene signature.

To further investigate the role of the Gene Signature (GS) in predicting a response to MAGE-A3 immunotherapeutic the PREDICT study was conducted in 49 centers in Europe and the United States on advanced melanoma patients. Because of frequent rapid progression in M1b-c melanoma, patients naive to previous systemic treatment with non-resectable stage IIIB-C and IV-M1a melanoma were included. This phase II study was not controlled, as placebo administration is unethical in this population and no highly effective treatment was available at the time of study design.

The OS of MAGE-A3-positive patients with unresectable stage IIIB-C/IV-M1a demonstrated an overall 1-year OS rate of 83.5\%. 1-year OS rates did not change when stratified in GS- and GS+ patients, indicating that in this study, GS was not predictive of outcome. Unexpectedly, the objective response rate was lower in this study than in other studies carried out in the same setting with the MAGE-A3 immunotherapeutic.

The MAGE 3 vaccine will probably no more undergo clinical investigations in the future, both in the adjuvant or therapeutic settings.

\section{BEVACIZUMAB}


Since Bevacizumab has reported to have some activity in patients with advanced melanoma, it has been valuated in the adjuvant setting. In a phase III multicenter trial conducted in the United Kingdom, 1,343 patients with resected stage IIB, IIC, or III disease were randomly assigned to receive 1 year of bevacizumab treatment $(7.5 \mathrm{mg} / \mathrm{kg}$ every 3 weeks $)$ or to observation. ${ }^{39}$ Results showed no significant difference in OS at a median follow-up of 25 months, (HR 0.97; 95\% CI, $0.78-1.22 ; \mathrm{p}=.76$ ). However, there was a significant increase in the DFS (1-year and 2-year disease-free rates $77 \%$ vs. $70 \%$ and $59 \%$ vs. $57 \%$, respectively; HR $0.83 ; 95 \%$ CI, $0.70-0.98$ ). Interpretation of the trial and the potential role of bevacizumab will require further follow-up to assess the 5-year OS rate. Until then, the use of bevacizumab in the adjuvant setting for patients with advanced melanoma is not recommended.

\section{RADIATION THERAPY AS ADJUVANT TREATMENT}

Adjuvant radiation treatment following CLND in the melanoma patient population has been suggested and investigated in order to gain regional control and consequently to improve survival ${ }^{40}$. Disease-free, survival rates and complications drive important issues on the therapeutic post CLND discussions. Historically, melanoma has been thought to be a relatively radioresistant tumour. Nowadays, radiation delivered according to the hypofractionated schedule is the most used, although there are no data to confirm that this schedule improves the therapeutic impact. Almost all the reviewed studies were retrospective, which could have led to an underestimation of the true incidence of the treatment toxicity and morbidity. Improved Loco regional control, but not OS has been reported when performing adjuvant radiotherapy after CLND for metastases of melanoma ${ }^{41}$. A recent study is describing an improvement in a subgroup of patients with a particular gene expression signature who would probably benefit from adjuvant radiotherapy ${ }^{42}$. The available data indicate the need for improved regional control rates in patients with extranodal extension, multiple involved nodes (more than three) and patients with large involved nodes (larger than $3 \mathrm{~cm})^{43}$. The complications seem manageable and consist mainly of fibrosis and edema. This treatment is mentioned in most guide lines where a discussion case by case is suggested after CLND. 


\section{TRIAL IN RESECTED STAGE II}

\section{NEW DRUGS IN STAGE III}

It is well known that T-cell responses are regulated through a complex balance of inhibitory and activating signals and that the tumour itself can dysregulate these pathways, leading therefore to an impairment of the immune system activities. The relevant new concept that was developed following the failure of cytokine-based immunotherapy and the increasing evidence of the clinical activity of different target therapies in several cancer types was constituted by the potential of targeting these inhibitory and activating immunological synapses as a new tool to promote the immune response ${ }^{44}$. Until now, two main types of immune modulating drug antibodies have been developed and used in the treatment of advanced metastatic melanoma, the first targeting the CTLA-4 antigens, the other the PD-1/PD-L1 pathway (Table1).

\subsection{IPILIMUMAB IN STAGE III}

The European Organisation for Research and Treatment of Cancer (EORTC) concluded the 18071 study on adjuvant ipilimumab versus placebo after complete resection of high-risk stage III melanoma patients ${ }^{45}$. It is the first trial with an immune checkpoint inhibitor used postoperatively after lymph node dissection, showing significant improvement in recurrence-free survival and overall survival (5-year RFS rates $40.8 \%$ vs. $30.3 \%$ and 5-year OS rates $65.4 \%$ vs. $54.4 \%$, respectively).

Despite clear benefits in reduction of risk of death, the use of adjuvant ipilimumab has not reached a global use: only FDA has approved the drug, while EMA did not activate the discussion on the same clinical indication. Considering the significant adverse event rates (grade 3-4 immune-related adverse events occurred in $41.6 \%$ of patients treated with ipilimumab as compared to $2.7 \%$ in placebo arm), resulting in only $42 \%$ of patients receiving more than four 
doses of ipilimumab, the survival benefit of ipilimumab over placebo was generally consistent across subgroups. This benefit was observed not only in patients with microscopic involvement in the SLN but also in patients with macroscopic or palpable nodes. Similarly, in contrast to interferon alfa, for which ulceration is the overriding determinant of activity, ipilimumab prolonged survival among patients with nonulcerated melanoma and among those with ulcerated melanoma and with no difference in terms of metastatic nodal involvement. The main topic for discussion has to be the dosage for IPI adjuvant administration: the EORTC study was designed to propose the same dosage of $10 \mathrm{mg} / \mathrm{m}^{2}$ for 3 years following the very first findings and study results in advanced melanoma ${ }^{46}$, but later the dosage of $3 \mathrm{mg} / \mathrm{m}^{2}$ was defined as efficacy as the higher dosage in this more advanced melanoma patients setting, so it appears irrational to approve for adjuvant use a drug that should be used at a more toxic, prolonged and expensive schedule than the same for an advanced disease indication.

In conclusion, it should be acknowledged that considering the low number of patients who had received the induction and mantainance phase of ipilimumab in the trial, the severe toxicity in a large number of patients, plus the lack of difference in DFS between the two dosage ( $3 \mathrm{mg} / \mathrm{kg}$ and the $10 \mathrm{mg} / \mathrm{kg}$ ) reported in the ASCO abstract ${ }^{47}$ (cit) had raised doubts on the usage of this drug as potential new candidate worldwide as adjuvant therapy in melanoma.

\subsection{ANTI PD-1 IN STAGE III}

Anti PD1 have beenapproved as first line treatmetn for metastatic melanoma.

Nivolumab and ipilimumab value in the adjuvant setting has been evaluated in a randomized, double-blind, phase 3 trial, randomly assigned 906 patients ( $\geq 15$ years of age) who were undergoing complete resection of stage IIIB, IIIC, or IV melanoma to receive an intravenous infusion of either nivolumab at a dose of $3 \mathrm{mg}$ per kilogram of body weight every 2 weeks (453 patients) or ipilimumab at a dose of $10 \mathrm{mg}$ per kilogram every 3 weeks for four doses and then every 12 weeks (453 patients) ${ }^{48}$. The period of treatment was up to 1 year or until disease recurrence, a report of unacceptable toxic effects, or withdrawal of consent. The 12-month rate of RFS primary endpoint was $70.5 \%$ in the nivolumab group and $60.8 \%$ in the ipilimumab group. Grade 3 or 4 adverse events that investigators deemed to be related to a trial drug were reported in $14.4 \%$ of the patients in the nivolumab group and in $45.9 \%$ of those in the ipilimumab group. There were 2 deaths $(0.4 \%)$ from toxic effects (marrow aplasia and colitis, both of which 
occurred more than 100 days after the last dose) in the ipilimumab group and no treatmentrelated deaths in the nivolumab group. The pathway for approval is under way through the regulatory agencies Worldwide (a part from the USA) and they will be approved by the time this article will be published.

Concerning Pembrolizumab, Keynote 054 tried to assess whether post-resection adjuvant therapy with pembrolizumab improves recurrence-free survival (RFS) as compared to placebo for highrisk participants with melanoma (Stage IIIA [ $>1 \mathrm{~mm}$ metastasis], IIIB and IIIC). Participants were stratified for stage of disease and region and then were randomly assigned to receive either pembrolizumab or placebo. 1019 stage IIIA-C melanoma patients were enrolled in the study. ${ }^{49}$

Patients were randomized to $200 \mathrm{mg}$ of pembrolizumab $(\mathrm{n}=514)$ or placebo $(\mathrm{n}=505)$ intravenously every 3 weeks for a total of 18 doses (approximately 1year) or until disease recurrence or unacceptable toxicity. Regarding - BRAF status, 40.9\% had a V600E or V600K mutation, $6.8 \%$ had another mutation, $45.3 \%$ were wild-type, and the status was unknown for $7.0 \%$. The primary endpoint (RFS rate) was $71.4 \%$ in pembrolizumab arm, (CI: 95\%, 66.8-75.4) versus 53.2\% in placebo arm (CI: 95\%, 47.9-58.2). An RFS benefit with the PD-1 inhibitor was observed across patients with either stage IIIA, IIIB, or IIIC disease. Grade 3-5 treatment-related adverse events occurred in $14.7 \%$ of the pembrolizumab arm versus $3.4 \%$ of the placebo group. The 1-year RFS rate was 77.1\% in the PD-L1-positive group, (CI: 95\%, 72.7-80.9) in the active arm and 62.6\% (CI: 96\%, 57.7-67.0) in the placebo one (HR, 0.54; CI: 95\%, 0.42-0.69; P < $.001)$. The 18 -month RFS rates were $74.2 \%$ versus $54.5 \%$, respectively.

Among PD-L1-negative patients, the 1-year RFS rates were 72.2\% (CI: 95\%, 58.6-82.0) in the pembrolizumab arm versus 52.2\% (CI:95\%, 38.2-64.5) in the placebo group (HR, 0.47; CI:95\%, $0.26-0.8 ; \mathrm{P}=.01)$. The 18 -month RFS rates were $60.6 \%$ versus $52.2 \%$, respectively [37].

In BRAF V600E positive patients, the 1-year RFS rate was $72.5 \%$ with pembrolizumab versus 58.6\% with placebo (HR, 0.57; CI: 99\%, 0.37-0.89; P = .0009). The 18-month RFS rates were $69.2 \%$ versus $52.4 \%$, respectively [37].

On the other hand, among BRAF wild-type patients, the 1-year RFS rate was $73.0 \%$ with pembrolizumab versus $59.7 \%$ with placebo (HR, 0.64; CI: 99\%, 0.42-0.96; $\mathrm{P}=.0039$ ). The $18-$ month RFS rates were $66.7 \%$ versus $48.8 \%$, respectively. 
The SWOG S1404 is a Phase III trial comparing high-dose IFN $\alpha$ with pembrolizumab (at doses of $200 \mathrm{mg}$ ) in patients with high-risk resected melanoma (stages III A-C and IV with no evidence of disease) for 52 weeks ${ }^{50}$. Primary outcomes include OS and RFS (ClinicalTrials.gov number: NCT02506153). This trial will offer new elements regarding the adjuvant treatment topic in melanoma.

\subsection{BRAF INHIBITORS IN STAGE III}

Approximately $50 \%$ of melanoma tumors have an activating mutation in the BRAF oncogene, which results in the constitutive activation of the MAP kinase signaling pathway ${ }^{51}$. Potent selective antagonists of mutant BRAF (vemurafenib and dabrafenib) are regarded as standard of care for metastatic BRAF mutant melanoma ${ }^{52} 53$ Of at least as much clinical importance, however, is the fact that approximately $90 \%$ of patients whose cancers carry the BRAF mutation have some tumor shrinkage with targeted inhibitors and that these responses occur very rapidly. This rapid response rate can provide palliative relief for patients with significant tumor-related symptoms but usually lasts 6 to 9 months when often a fast recurring disease appears and other therapies need to be considered 5455565758 .

Activated BRAF phosphorylates MEK, the next downstream target in the MAP kinase pathway. Trametinib and Cobimetinib are selective MEK inhibitors and both have been shown to improve median progression free and overall survival in comparison to chemotherapy in BRAF mutant melanoma ${ }^{6264,59}$ Dual inhibition of both BRAF and MEK pathways in BRAF mutant disease is now proven to be significantly more potent than single agent inhibition in advanced melanoma patients.

\subsection{Single drug adjuvant treatment with BRAF inhibitor}

The BRIM 8 study was designed as a double bling placebo controlled study of adjuvant vemurafenib in patients with completed resected BRAF V600+ mutant melanoma at high risk for 
recurrence. Results from this study showed a clinical benefit of the treatment arm in stages IICIIIB ( $\mathrm{HR}=0.54, \mathrm{p}<0.001)$ but not for stage IIIC $(\mathrm{HR}=0.80, \mathrm{p}=0.26)$, when survival estimates curves are not significantly different. Considering the study design, the primary DFS endpoint was not met in patients with resected stage IIIC $B R A F^{\mathrm{V} 600+}$ melanoma, although one year of adjuvant vemurafenib showed a numerical DFS benefit in patients with resected stage IIC/IIIA/IIIB disease.

The role of BRAF inhibitor alone should be put in the context of recently reported trials. The placebo-controlled COMBI-AD study showed that combination adjuvant treatment with a MEK and a BRAF inhibitor is able to reduce the risk of recurrence in patients with resected stage III $B R A F^{\mathrm{V} 600+}$ melanoma (HR 0.47, 95\% CI 0·39-0.58; $<<0.001$ ) (referenza). In addition, a recent head-to-head adjuvant study (CheckMate 238) comparing nivolumab versus ipilimumab in patients with resected stage IIIB/C-IV melanoma showed that nivolumab significantly reduced the rate of recurrence or death (HR $0 \cdot 65,95 \%$ CI $0 \cdot 51-0 \cdot 83, \mathrm{p}<0.001)$ with a lower incidence of grade $3 / 4$ events $(25 \cdot 4 \%$ vs $55 \cdot 2 \%)$ and AE-related discontinuation rate $(9 \cdot 7 \% v s$ $42 \cdot 6 \%)^{56}$. Based on these results, it is clear that combination adjuvant treatment with BRAF and MEK inhibitors or single-agent nivolumab provide more favourable DFS and survival outcomes in patients with melanoma who are at high risk of recurrence. Although, we cannot exclude a role for single-agent BRAF inhibitors within certain disease substages (IIC) of this patient population, although there are no ongoing or planned studies to explore this.

The treatment with single drug BRAF inhibitor/s side effects may be one important aspect to consider for not approving the treatment in the adjuvant settings: these include a variety of different effects with the majority occurring on the skin and appendage ${ }^{60,61,62}$. The most common adverse events recorded in the BRIM-3 registration trial included arthralgia, fatigue, nausea, rashes, photosensitivity and cutaneous squamous cell carcinoma ( $\mathrm{CSCC}$ ) or keratoacanthoma $(\mathrm{KA})^{63}$. The dose was modified or interrupted due to adverse events in $38 \%$ of patients treated 
with vemurafenib and permanently discontinued in only $7 \%$ of the patients treated. When transferring these treatment/s from advanced melanoma patients into a concept of adjuvant therapy all these aspects have to be considered: globally a high percentage of patients would not benefit from any adjuvant therapy as already cured by surgery: a treatment with important side effects and an alteration of the quality of life with no selection on the patients who may really benefit form a treatment is not going to be easily accepted by most patients.

\subsection{Combination target therapy}

The results of the fase III COMBI-AD adjuvant study Adjuvant Dabrafenib plus Trametinib in Stage III BRAF-Mutated Melanoma have been positive and will certainly modify the clinical practice in the next future. This was a double-blind, placebo-controlled, phase 3 trial, enrolling 870 patients with completely resected, stage III melanoma with BRAF V600E or V600K mutations that were randomly assigned to receive oral dabrafenib at a dose of $150 \mathrm{mg}$ twice daily plus trametinib at a dose of $2 \mathrm{mg}$ once daily (combination therapy, 438 patients) or two matched placebo tablets (432 patients) for 12 months. Primary end point was the RFS and showed the $58 \%$ in the combination-therapy group versus $39 \%$ in the placebo arm group $(\mathrm{P}<0.001)$. The main secondary end point was overall survival and formally did not reach the forecasted results but in any case the 3-year overall survival rate was $86 \%$ in the combination-therapy group and $77 \%$ in the placebo group $(\mathrm{P}=0.0006)$, instead of the prespecified interim analysis boundary of $\mathrm{P}=0.000019$. The safety profile of dabrafenib plus trametinib was consistent with that observed with the combination in patients with metastatic melanoma. ${ }^{64}$ The pathway for approval in the adjuvant setting is under way through the regulatory agencies Worldwide and might be approved by the time this article will be published.

\section{FUTURE DEVELOPMENT}

The introduction of the adjuvant setting would change our way of looking and studying the disease, swithching from the main goal that was disease free survival to a longer vision of the disease. Already in the era of immunotherapy we have assisted to longer melanoma specific survival even despite quick relapse free survival or even progression after first relapse. 
The use of PD-1 inhibitors in the adjuvant setting has been a major discussion topic in last years. Some of the challenges of assessing these drugs in the adjuvant setting include choosing a comparator arm, due to the standard of care being unclear, patient selection, unexpected toxicity, and deciding how long to treat the patient. Another challenge is presented by the selection of a meaningful primary endpoint, whether that is OS or RFS.. An interesting question on the role of adjuvant therapies has to be raised: is it important to start a treatment in the adjuvant setting which means to treat a robust percentage of patients who are already potentially cured with surgery or, at the end, the survival obtained will be the same in case patients are treated only after recurrence of their disease?

There are some data suggested that due to the primed immune system you get more toxicity in the adjuvant setting than in the advanced disease setting [39]. A number of important questions have found response by trials mentioned above, but remain many other questions that need to be settled regarding the use of anti-PD-1 blockade in the adjuvant setting. For example if PD-L1 expression in resected melanoma tumors serve as a biomarker for successful adjuvant treatment, and if there are other novel biomarkers that could be used in order to differentiate between patients who will derive the most benefit from treatment, without exposing

to unnecessary ineffective and toxic treatment this population. In conclusion, a major advantage of immunotherapy is the possibility to discontinue treatment and maintain antitumor responses. The immunological 'memory' induced by the immunotherapy agent offers the potential for longlasting, possibly life-long, therapeutic responses.

For sure the identification of the patietns is a matter of debate and will be one of the first issue to be solved. The American Joint Committee on Cancer (AJCC) has reported in its $8^{\text {th }}$ edition a melanoma-specific survival (MSS) for all stage sub- groups higher than those reported in the seventh edition.

The higher survival of patients in the more contemporary cohort examined is likely a consequence of the widespread use of sentinel lymph node (SLN) biopsy; the requirement of SLN biopsy for patients with T2 through T4 primary melanoma to be included in AJCC staging; 
and, to a lesser extent, newer imaging technologies that improve the detection of clinically occult metastatic disease.

Despite this, there is still a marked prognostic heterogeneity within the same stage and a prognostic hierarchy between the different stages seems to lack.

Within stage III, a new subgroup (stage IIID) has been added with respect to the previous edition. Consequently, the prognosis of stage IIIA patients has improved, while a higher risk subgroup of stage III patient has been identified.so the Anyway, no one of these trials has focused on stage IIC patients who may deserve of adjuvant therapy as well as stage III ones (Fig. 2). If their results would be the base for adjuvant therapy indications, there is the risk of an unfair limitation to the clinical practic Anyway, no one of these trials has focused on stage IIC patients who may deserve of adjuvant therapy as well as stage III ones (Fig. 2). If their results would be the base for adjuvant therapy indications, there is the risk of an unfair limitation to the clinical practice

Combination therapies are at the moment under consideration .

Programmed cell death-1 (PD1) is an immune inhibitory receptor expressed by activated T and $\mathrm{B}$ cells that binds to the two known ligands PDL1 and PDL2. PDL1 is expressed by a wide variety of tissues, and also on human tumors, including melanoma. When PD1 binds to its ligands, it negatively regulates T-cell function ${ }^{65}$. This mechanism is used by several tumors to escape the immune system control. According to recent studies IFN could regulate PD1/PDL1-2 expression resulting in a controversial pro-tumor escape effect . In fact, CD8+ T cells matured in the presence of IFN- $\alpha$ showing higher levels of PD1 and a relatively poor ability to inhibit tumor growth efficiently ${ }^{66}$. Moreover, IFN- $\alpha$ has been reported to increase PDL1 cellular expression on hepatocytes ${ }^{67}$, and IFN- $\gamma$ on tumor cells ${ }^{68}$. These lines of evidence support the hypothesis that IFN- $\alpha$-mediated anti-tumor activity would be significantly enhanced through PD-1 blockade. The combination of the anti-PD1 pembrolizumab and pegylated IFN- $\alpha 2 b$ was recently tested in a phase I clinical trial and was well tolerated with no dose limiting toxicities and mostly grade 1 adverse events. Enrolled patients were affected by recurrent inoperable stage III and IV melanoma and previous treatments included adjuvant IFN, vemurafenib, chemotherapy, and radiotherapy. Six out of 12 patients were evaluated for clinical responses at week 12 with 1 
complete response, 4 stable disease, and 1 progressive disease with mixed clinical responses ${ }^{69}$. In stage II, we do not have studies at the moment with new adjuvant treatments, although those patients represent a big percentage of the total amount of potential candidates to an adjuvant treatment. In the latter, to decrease DFS while increasing the OS will globally impact even more than in stage III on the global survival of melanoma patients.

Not completely similarly to advanced disease we shall now face a new difficult decision to be taken at least in half of the patients: which therapy to adopt in the adjuvant setting for patients presenting BRAF mutation? In metastatic disease we shall always have the chance to propose both treatments and the main issue is to decide which to propose as first line, and in this advance disease patients population the choice is usually justified or motivated on the basis of an empiric feeling: offer a target therapy to patients with bulky, aggressive disease, and a immunotherapy to more chronic low aggressive metastatic progression of melanoma. Can we apply the same philosophy to the adjuvant setting? Probably not, but more than this, in the adjuvant setting we shall be able to offer only one treatment schedule, so the choice will be at this stage more a patient to patient discussion then a decision driven from clinical or biological issues: patients with easy accessibility to the hospital willing to receive ev. Injection every 3 weeks will be offered immunotherapy, while older, less geographically accessible patients with less sun exposure risk may prefer the oral approach of target therapy.

The large spectrum of prognosis in stage III patients is opening a huge request of biomarker to evaluate the risk of progression and to identify whom out of all the patients will develop a disease progression. Many clinical and pathologic prognostic factors have been evaluated. Clinically age and phenotype (like mole count) has been demonstrated to play a role in survival in positive SLN patients ${ }^{70}$. Pathologic features of primary like Breslow thickness, ulceration, mitoses and regression have been associated to prognosis as well as the number of lymph node excised and the number of positive ones ${ }^{5,6,71}$. Genetic biomarkers are now under evaluation. The urgent need of biomarker has increased after the discovery of efficacy in the adjuvant therapy in terms of increasing survival. To be able to detect which patients are at risk of progression and so to candidate only those ones to adjuvant therapy would be the next goal of melanoma research. 
Last topic under study will be the concept of neoadjuvant therapies: this approach could be proposed in different scenarios i.e. for stage III palpable nodal disease and for stage IV melanoma patients. Years ago the only possibly effective therapy was surgery. Now 2 studies demonstrate that surgery is no more the gold standard and new hypothesis are under investigation from the medical point of view. In stage IV on the contrary, surgery could be seen as a confirmation of efficacy of medical treatments where both a complete response or a partial response have been reached, offering to patients the opportunity of interrupting their medical treatments once the condition of no evidence of residual disease has been confirmed.

\section{CONCLUSION}

Melanoma clinical research during the last 10 years has driven the most important changes in treatment approaches seen in oncology since ever. From being an orphan and neglected disease it has moved to the most pioneering tumor from which so many other cancer types are learning and developing new strategies ${ }^{72}$. Advanced disease has demonstrated an impressive treatment efficacy improvement passing from 5\% to more then 50\% survival benefit at 5 years. From these results a new set of trials have been developed in the adjuvant setting and the results of the first studies have been recently offered to the scientific community to become common practice as soon as regulatory agencies will permit their clinical use.

\section{References}

${ }^{1}$ Ribero S, Glass D, Bataille V. Genetic epidemiology of melanoma. Eur J Dermatology. 2016;26(4):335-9.

${ }^{2}$ Balch CM, Gershenwald JE, Soong SJ, et al. Final version of 2009 AJCC melanoma staging and Classification. J Clin Oncol 2009; 27(36):6199-6206.

${ }^{3}$ Gershenwald JE, Scolyer RA, Hess KR, Sondak VK, Long GV, Ross MI,et al.; for members of the American Joint Committee on Cancer Melanoma Expert Panel and the International Melanoma Database and Discovery Platform.Melanoma staging: Evidence-based changes in the 
American Joint Committee on Cancer eighth edition cancer staging manual. CA Cancer J Clin. 2017 Nov;67(6):472-492.

${ }^{4}$ Grob JJ, Schadendorf D, Lorigan P, Ascierto P, Larkin J, Nathan P, et al.Eighth American Joint Committee on Cancer (AJCC) melanoma classification: Let us reconsider stage III. Eur J Cancer. 2017 Dec 7. pii: S0959-8049(17)31431-4.

5 Testori A, De Salvo G, Montesco M, Trifiro' G, Mocellin S, Landi G, et al. Clinical considerations on sentinel node biopsy in melanoma from italian multicentric study on 1,313 patients (SOLISM-IMI) - Ann Surg Oncol, 2009 Jul;16(7):2018-27. doi: 10.1245/s10434-0080273-8. Epub 2009 Jan 9.

${ }^{6}$ Mandalà M, Galli F, Cattaneo L, Merelli B, Rulli E, Ribero S, et al. Mitotic rate correlates with sentinel lymph node status and outcome in cutaneous melanoma greater than 1 millimeter in thickness: A multi-institutional study of 1524 cases. J Am Acad Dermatol. 2016;

7 Quaglino P, Ribero S, Osella-Abate S, Macri L, Grassi M, Caliendo V, et al. Clinicopathologic features of primary melanoma and sentinel lymph node predictive for non-sentinel lymph node involvement and overall survival in melanoma patients: a single centre observational cohort study. Surg Oncol 2011; 20 (4): 259-264

8 Faries MB, Thompson JF, Cochran AJ, Andtbacka RH, Mozzillo N, Zager JS, et al. Completion Dissection or Observation for Sentinel Node Metastasis in Melanoma Engl J Med 2017;376:2211-22. DOI: 10.1056/NEJMoa1613210

${ }^{9}$ Leiter U, Stadler R, Mauch C, Hohenberger W, Brockmeyer N, Berking C, et al. Complete lymph node dissection versus no dissection in patients with sentinel lymph node biopsy positive melanoma (DeCOG-SLT): a multicentre, randomised, phase 3 trial. Lancet Oncol. 2016 Jun;17(6):757-767. doi: 10.1016/S1470-2045(16)00141-8

${ }^{10}$ Pasquali S, Mocellin S, Mozzillo N, Maurichi A, Quaglino P, Borgognoni L et al.Nonsentinel lymph node status in patients with cutaneous melanoma: results from a multi-institution prognostic study. J Clin Oncol. 2014 Mar 20;32(9):935-41

${ }^{11}$ Sandro P, Andrea M, Nicola M, Simone M, Giuseppe M, Lorenzo B,et al. Lymph-Node Ratio in Patients with Cutaneous Melanoma: A Multi-Institution Prognostic Study. Ann Surg Oncol. $2015 \mathrm{Jul} ; 22(7): 2127-34$. 
12 Rossi CR, Mocellin S, Campana LG, Borgognoni L, Sestini S, Giudice G, et al. Prediction of Non-sentinel Node Status in Patients with Melanoma and Positive Sentinel Node Biopsy: An Italian Melanoma Intergroup (IMI) Study. Ann Surg Oncol. 2017 Oct 24.

13 Flaherty LE, Othus M, Atkins MB, Tuthill RJ, Thompson JA, Vetto JT et al. Southwest Oncology Group S0008: A phase III trial of high-dose interferon alfa-2b versus cisplatin, vinblastine, and dacarbazine DTIC, plus interleukin-2 and interferon in patients with high-risk melanoma - an Intergroup Study of Cancer and Leukemia Group B, Children's Oncology Group, Eastern Cooperative Oncology Group, and Southwest Oncology Group. J Clin Oncol. 2014 Nov 20;32(33):3771-8

14 Figlin RA, Thompson JA, Bukowski RM, Vogelzang NJ, Novick AC, Lange P, et al. Multicenter, randomized, phase III trial of $\mathrm{CD} 8(+)$ tumor-infiltrating lymphocytes in combination with recombinant interleukin-2 in metastatic renal cell carcinoma. J Clin Oncol. 1999;17:2521-9.

${ }^{15}$ Veronesi U, Adamus J, Aubert C, Bajetta E, Beretta G, Bonadonna G, et al. A Randomized Trial of Adjuvant Chemotherapy and Immunotherapy in Cutaneous Melanoma. N Engl J Med $1982 ; 307: 913-916$

16 Agarwala SS, Neuberg D, Park Y, Kirkwood JM. Mature results of a phase III randomized trial of bacillus Calmette-Guerin (BCG) versus observation and BCG plus dacarbazine versus BCG in the adjuvant therapy of American Joint Committee on Cancer Stage I-III melanoma (E1673) A trial of the Eastern Cooperative Oncology Group. Cancer 2004 Apr 15;100(8):1692-8.

17 Faries MB, Mozzillo N, Kashani-Sabet M, Thompson JF, Kelley MC, DeConti RC, et al. Long-Term Survival after Complete Surgical Resection and Adjuvant Immunotherapy for Distant Melanoma Metastases. Ann Surg Oncol (2017) 24:3991-4000

18 Zitvogel L, Galluzzi L, Kepp O, Smyth MJ, Kroemer G. Type I interferons in anticancer immunity. Nat Rev Immunol. 2015 Jul;15(7):405-14.

19 Raig ET, Jones NB, Varker KA, Benniger K, Go MR, Biber JL, et al. VEGF secretion is inhibited by interferon-alpha in several melanoma cell lines. J Interferon Cytokine Res. 2008 Sep;28(9):553-61.

20 Palmer KJ, Harries M, Gore ME, Collins MK.Interferon-alpha (IFN-alpha) stimulates antimelanoma cytotoxic T lymphocyte (CTL) generation in mixed lymphocyte tumour cultures (MLTC). Clin Exp Immunol. 2000 Mar;119(3):412-8. 
${ }^{21}$ Kirkwood JM, Strawderman MH, Ernstoff MS, Smith TJ, Borden EC, Blum RH. Interferon alfa-2b adjuvant therapy of high-risk resected cutaneous melanoma: the Eastern Cooperative Oncology Group Trial EST 1684. J Clin Oncol. 1996 Jan;14(1):7-17.

22 Hauschild A, Weichenthal M, Rass K, Linse R, Ulrich J, Stadler R,et al. Prospective randomized multicenter adjuvant dermatologic cooperative oncology group trial of low-dose interferon alfa- $2 b$ with or without a modified high-dose interferon alfa- $2 b$ induction phase in patients with lymph node-negative melanoma. J Clin Oncol. 2009 Jul 20;27(21):3496-502.

${ }^{23}$ Kirkwood JM, Ibrahim JG, Sondak VK, Richards J, Flaherty LE, Ernstoff MS, et al. Highand low-dose interferon $\alpha-2 b$ in high-risk melanoma: first analysis of Intergroup Trial E1690/S9111/C9190. J. Clin. Oncol., 18: 2444-2458, 2000

${ }^{24}$ Hauschild A, Weichenthal M, Rass K, Linse R, Berking C, Böttjer J et al.Efficacy of low-dose interferon \{alpha\} 2a 18 versus 60 months of treatment in patients with primary melanoma of $>=$ $1.5 \mathrm{~mm}$ tumor thickness: results of a randomized phase III DeCOG trial. J Clin Oncol. 2010 Feb 10;28(5):841-6. doi: 10.1200/JCO.2009.23.1704.

${ }^{25}$ Eggermont AM, Suciu S, Rutkowski P, Kruit WH, Punt CJ, Dummer R, et al. Long term follow up of the EORTC 18952 trial of adjuvant therapy in resected stage IIB-III cutaneous melanoma patients comparing intermediate doses of interferon-alpha-2b (IFN) with observation: Ulceration of primary is key determinant for IFN-sensitivity. Eur J Cancer. 2016 Mar;55:111-21.

${ }^{26}$ Mocellin S, Pasquali S, Rossi CR, Nitti D. Interferon alpha adjuvant therapy in patients with high-risk melanoma: a systematic review and meta-analysis.J Natl Cancer Inst. 2010 Apr 7;102(7):493-501.

27 Ives NJ, Suciu S, Eggermont AMM, Kirkwood J, Lorigan P, Markovic SN, et al. Adjuvant interferon- $\alpha$ for the treatment of high-risk melanoma: An individual patient data meta-analysis. Eur J Cancer. 2017 Sep;82:171-183.

${ }^{28}$ Eggermont AM, Suciu S, Testori A, Santinami M, Kruit WH, Marsden J, et al. Long-term results of the randomized phase III trial EORTC 18991 of adjuvant therapy with pegylated interferon alfa-2b versus observation in resected stage III melanoma. J Clin Oncol. 2012 Nov $1 ; 30(31): 3810-8$

${ }^{29}$ Eggermont AM, Suciu S, Testori A, Kruit WH, Marsden J, Punt CJ, et al. Ulceration and stage are predictive of interferon efficacy in melanoma: results of the phase III adjuvant trials EORTC 18952 and EORTC 18991. Eur J Cancer. 2012 Jan; 48(2):218-25. 
${ }^{30}$ Eggermont AM, Suciu S, Santinami M, Testori A, Kruit WH, Marsden J, et al. Adjuvant therapy with pegylated interferon alfa- $2 \mathrm{~b}$ versus observation alone in resected stage III melanoma: final results of EORTC 18991, a randomised phase III trial. Lancet 2008;372:11726.

${ }^{31}$ Stockwin LH, McGonagle D, Martin IG, Blair GE.Dendritic cells: immunological sentinels with a central role in health and disease. Immunol Cell Biol. 2000 Apr;78(2):91-102.

${ }^{32}$ Nencioni A, Grünebach F, Schmidt SM, Müller MR, Boy D, Patrone F, et al.The use of dendritic cells in cancer immunotherapy. Crit Rev Oncol Hematol. 2008 Mar;65(3):191-9.

33 Boudewijns S, Bol KF, Schreibelt G, Westdorp H, Textor JC, van Rossum MM et al., Adjuvant dendritic cell vaccination induces tumor specific immune responses in the majority of stage III melanoma patients. Oncoimmunology. 2016 May 31;5(7):e1191732.

${ }^{34}$ Schumacher TN, Schreiber RD. Neoantigens in cancer immunotherapy. Science. 2015 Apr 3; 348(6230):69-74.

${ }^{35}$ Carreno BM, Magrini V, Becker-Hapak M, Kaabinejadian S, Hundal J, Petti AA,et al. Cancer immunotherapy. A dendritic cell vaccine increases the breadth and diversity of melanoma neoantigen-specific T cells.Science. 2015 May 15; 348(6236):803-8

${ }^{36}$ Delamarre L, Mellman I, Yadav M. Cancer immunotherapy. Neo approaches to cancer vaccines. Science. 2015 May 15; 348(6236):760-1.

${ }^{37}$ Eggermont AM, Suciu S, Rutkowski P, Marsden J, Santinami M, Corrie P, et al. Adjuvant Ganglioside GM2-KLH/QS-21 Vaccination Versus Observation After Resection of Primary Tumor $>1.5 \mathrm{~mm}$ in Patients With Stage II Melanoma: Results of the EORTC 18961 Randomized Phase III Trial.J Clin Oncol. 2013 Oct 20;31(30):3831-7

${ }^{38}$ Saiag P, Gutzmer R, Ascierto PA, Maio M, Grob JJ, Murawa P, et al. Prospective assessment of a gene signature potentially predictive of clinical benefit in metastatic melanoma patients following MAGE-A3 immunotherapeutic (PREDICT). Ann Oncol. 2016 Oct;27(10):1947-53.

${ }^{39}$ Corrie PG, Marshall A, Dunn JA, Middleton MR, Nathan PD, Gore M, et al. Adjuvant bevacizumab in patients with melanoma at high risk of recurrence (AVAST-M): preplanned interim results from a multicentre, open-label, randomised controlled phase 3 study. Lancet Oncol. 2014 May;15(6):620-30. 
40 Bastiaannet E, Beukema JC, Hoekstra HJ.Radiation therapy following lymph node dissection in melanoma patients: treatment, outcome and complications. Cancer Treat Rev. 2005 Feb;31(1):18-26.

${ }^{41}$ Keenan LG, O'Sullivan S, Glynn A, Higgins M, Flavin A, Brennan S. Clinical review of treatment outcomes and patterns of failure with adjuvant radiotherapy in node-positive malignant melanoma. J Med Imaging Radiat Oncol. 2017 Apr;61(2):258-262. doi: 10.1111/17549485.12536. Epub 2016 Sep 25.

42 Strom T, Torres-Roca JF, Parekh A, Naghavi AO, Caudell JJ, Oliver DE et al. Regional Radiation Therapy Impacts Outcome for Node-Positive Cutaneous Melanoma. J Natl Compr Canc Netw. 2017 Apr;15(4):473-482.

43 Moncrieff MD, Martin R, O'Brien CJ, Shannon KF, Clark JR, Gao K, et al..Adjuvant postoperative radiotherapy to the cervical lymph nodes in cutaneous melanoma: is there any benefit for high-risk patients? Ann Surg Oncol. 2008 Nov;15(11):3022-7.

44 Ribero S, Longo C, Glass D, Nathan P, Bataille V. What is New in Melanoma Genetics and Treatment? Dermatology. 2016;232(3):259-64.

45 Eggermont AMM, Chiarion-Sileni V, Grob JJ, Dummer R, Wolchok JD, Schmidt H, et al. Prolonged Survival in Stage III Melanoma with Ipilimumab Adjuvant Therapy N Engl J Med 2016; 375:1845-1855November 10, 2016DOI: 10.1056/NEJMoa1611299

46 Weber J, Thompson JA, Hamid O, Minor D, Amin A, Ron I, et al. A randomized, doubleblind, placebo-controlled, phase II study comparing the tolerability and efficacy of ipilimumab administered with or without prophylactic budesonide in patients with unresectable stage III or IV melanoma.Clin Cancer Res. 2009 Sep 1;15(17):5591-8.

${ }^{47}$ Luke JJ, Is There an Optimal Dose of Ipilimumab in Melanoma? ASCO meeting 2017

48 Weber J, Mandala M, Del Vecchio M, Gogas HJ, Arance AM, Cowey CL, et al. Adjuvant Nivolumab versus Ipilimumab in Resected Stage III or IV Melanoma. N Engl J Med. 2017 Nov 9;377(19):1824-1835.

${ }^{49}$ Eggermont AMM, Blank CU, Mandala M, Long GV, Atkinson V, Dalle S, et al. Adjuvant pembrolizumab versus placebo in resected stage III melanoma. N Engl J Med 2018;378:1789-801.

50 Grossmann, KF Othus M, Tarhini AA, Patel SP, Moon J, Sondak VK, et al. SWOG S1404: a phase III randomized trial comparing standard of care adjuvant therapy to pembrolizumab in patients with high risk resected melanoma. ASCO annual meeting; 2017 (abstract e21032). 
${ }^{51}$ Davies H, Bignell GR, Cox C Stephens P, Edkins S, Clegg S, et al. Mutations of the BRAF gene in human cancer. Nature. 2002 Jun 27;417(6892):949-54.

52 Chapman PB, Hauschild A, Robert C, Haanen JB, Ascierto P, Larkin J, et al. Improved survival with vemurafenib in melanoma with BRAF V600E mutation. N Engl J Med. 2011 Jun 30;364(26):2507-16

53 Long GV, Trefzer U, Davies MA, Kefford RF, Ascierto PA, Chapman PB, et al. Dabrafenib in patients with Val600Glu or Val600Lys BRAF-mutant melanoma metastatic to the brain (BREAK-MB): a multicentre, open-label, phase 2 trial. Lancet Oncol. 2012 Nov;13(11):108795.

54 Das Thakur M, Salangsang F, Landman AS, e Sellers WR, Pryer NK, Levesque MP et al. Modelling vemurafenib resistance in melanoma reveals a strategy to forestall drug resistance. Nature. 2013 Feb 14;494(7436):251-5.

55 Trunzer K, Pavlick AC, Schuchter L, Gonzalez R, McArthur GA, Hutson TE, et al. Pharmacodynamic effects and mechanisms of resistance to vemurafenib in patients with metastatic melanoma. J Clin Oncol 2013;31:1767-74.

${ }^{56}$ Long GV, Stroyakovskiy D, Gogas H, Levchenko E, de Braud F, Larkin J, et al. Combined BRAF and MEK inhibition versus BRAF inhibition alone in melanoma. N Engl J Med. 2014 Nov 13;371(20):1877-88.

${ }^{57}$ Long GV, Stroyakovskiy D, Gogas H, Levchenko E, de Braud F, Larkin J et al. Dabrafenib and trametinib versus dabrafenib and placebo for Val600 BRAF-mutant melanoma: a multicentre, double-blind, phase 3 randomised controlled trial. Lancet. 2015 Aug 1;386(9992):444-51.

${ }^{58}$ Larkin J, Ascierto PA, Dréno B, Atkinson V, Liszkay G, Maio M et al. Combined vemurafenib and cobimetinib in BRAF-mutated melanoma. N Engl J Med. 2014 Nov 13;371(20):1867-76

${ }^{59}$ Flaherty KT, Robert C, Hersey P, Nathan P, Garbe C, Milhem M et al. Improved survival with MEK inhibition in BRAF-mutated melanoma. N Engl J Med. 2012 Jul 12;367(2):107-14.

${ }^{60}$ Sanlorenzo M, Choudhry A, Vujic I, Posch C, Chong K, Johnston K et al. Comparative profile of cutaneous adverse events: BRAF/MEK inhibitor combination therapy versus BRAF monotherapy in melanoma. J Am Acad Dermatol. 2014 Dec;71(6):1102-1109.

${ }^{61}$ Dika E, Ravaioli GM, Fanti PA, Piraccini BM, Lambertini M, Chessa MA,et al. 
Cutaneous adverse effects during ipilimumab treatment for metastatic melanoma: a prospective study. Eur J Dermatol. 2017 Jun 1;27(3):266-270. doi: 10.1684/ejd.2017.3023.

62 Dika E, Patrizi A, Ribero S, Fanti PA, Starace M, Melotti B, Sperandi F, Piraccini BM.

Hair and nail adverse events during treatment with targeted therapies for metastatic melanoma. Eur J Dermatol. 2016 Jun 1;26(3):232-9. doi: 10.1684/ejd.2016.2747.

63 Chapman PB, Hauschild A, Robert C, Haanen JB, Ascierto P, Larkin J, et al. Improved survival with vemurafenib in melanoma with BRAF V600E mutation. N Engl J Med 2011; 364 : 2507-16

64 Long GV, Hauschild A, Santinami M, Atkinson V, Mandalà M, Chiarion-Sileni V, et al. Adjuvant Dabrafenib plus Trametinib in Stage III BRAF-Mutated Melanoma. N Engl J Med. 2017 Nov 9;377(19):1813-1823.

${ }^{65}$ Riella LV, Paterson AM, Sharpe AH, Chandraker A. Role of the PD-1 pathway in the immune response. Am J Transplant. 2012 Oct;12(10):2575-87.

66 Rafique I, Kirkwood JM, Tarhini AA.Immune checkpoint blockade and interferon- $\alpha$ in melanoma. Semin Oncol. 2015 Jun;42(3):436-47.

67 Mühlbauer M, Fleck M, Schütz C, Weiss T, Froh M, Blank C, et al. PD-L1 is induced in hepatocytes by viral infection and by interferon-alpha and -gamma and mediates $\mathrm{T}$ cell apoptosis. J Hepatol. 2006 Oct;45(4):520-8.

${ }^{68}$ Abiko K, Matsumura N, Hamanishi J, Horikawa N, Murakami R, Yamaguchi K, et al. IFN- $\gamma$ from lymphocytes induces PD-L1 expression and promotes progression of ovarian cancer. $\mathrm{Br} \mathrm{J}$ Cancer. 2015 Apr 28;112(9):1501-9.

69 Davar D, Wang H, Chauvin JM, Sun Z, Pagliano O, Rose A, et al. Phase IB study of pembrolizumab (Pembro) and pegylated-interferon alfa-2b (Peg-IFN) in advanced melanoma (MEL). J Clin Oncol 2016 34:15_suppl, 9539-9539

${ }^{70}$ Ribero S, Davies JR, Requena C, Carrera C, Glass D, Rull R, et al. High naevus counts confer a favourable prognosis in melanoma patients. Int J Cancer. 2015 Oct 1;137(7):1691-8.

${ }^{71}$ Rossi CR, Mozzillo N, Maurichi a, Pasquali S, Quaglino P, Borgognoni L, et al. The number of excised lymph nodes is associated with survival of melanoma patients with lymph node metastasis. Ann Oncol . 2014;25(1):240-6. 
${ }^{72}$ Marconcini R, Spagnolo F, Stucci LS, Ribero S, Marra E, De Rosa F, et al. Current status and perspectives in immunotherapy for metastatic melanoma oncotarget in press 